\title{
A Rapid Approach for Fabricating Boronic Acid- Functionalized Plates for On-Probe Detection of Glycoprotein and Glycopeptide
}

\author{
Yu-Ching Liu ${ }^{1}$ and Chao-Jung Chen ${ }^{* 1,2}$ \\ ${ }^{1}$ Proteomics Core Laboratory, Department of Medical Research, China Medical University Hospital, Taichung 40402, Taiwan \\ ${ }^{2}$ Graduate Institute of Integrated Medicine, China Medical University, Taichung 40402, Taiwan
}

\begin{abstract}
We developed a rapid and simple approach without using complex mechanical or chemical protocols to fabricate boronic acid-functionalized plates for glycoprotein or glycopeptide enrichment and mass spectrometry (MS) analysis. By coating the boronic acid-functionalized silica particles on a polydimethylsiloxane (PDMS)-coated matrix-assisted laser desorption/ionization (MALDI) plate, these particles can form a firmly monolayer of particles on PDMS membrane for sample handling without peeling off. The boronic acid particles-coated PDMS plate (BP plate) was successfully applied to the enrichment of horseradish peroxidase (HRP) protein and their digested glycopeptides.
\end{abstract}

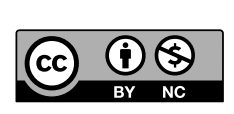

Copyright (c) 2017 Yu-Ching Liu and Chao-Jung Chen. This is an open access article distributed under the terms of Creative Commons Attribution License, which permits use, distribution, and reproduction in any medium, provided the original work is properly cited and is not used for commercial purposes.

Please cite this article as: Mass Spectrom (Tokyo) 2017; 6(2): S0063

Keywords: boronic acid, glycoprotein, MALDI-TOF, on-probe

(Received September 28, 2016; Accepted January 11, 2017)

\section{INTRODUCTION}

Protein glycosylation is the most common post-translational modification and involves in many important biological functions, e.g., protein folding, intracellular sorting, secretion, uptake, and cell recognition. ${ }^{1)}$ In mass spectrometry analysis, low-abundance glycoprotein or glycopeptide is usually further suppressed by the presence of abundant nonglycoprotein/non-glycopeptides; therefore, glycoprotein or glycopeptide enrichment is usually performed prior to MS ananlysis. Many methods have been developed for glycoprotein/glycopeptide enrichment, such as lectin affinity, ${ }^{2)}$ size exclusion, ${ }^{3)}$ hydrazide chemistry, ${ }^{4)}$ and hydrophilic interaction chromatography. ${ }^{5)}$

Recently, boronic acid-based chemical has been greatly used in the unbiased enrichment of $\mathrm{N}$ - and $\mathrm{O}$-glycoproteins/ glycopeptides through covalent binding with a 1,2-cis-diol of glycol-structure. Boronic acid has been immobilized on magnetic nanoparticles, mesoporous silica, polymer nanoparticles, and agarose resin that were for solid phase extraction (SPE) of glycoproteins/glycopeptides. However, SPE approaches may bring concerns of sample loss and time-consuming preparation process. In addition, miniaturized SPE approaches such as packed tips or microtiter SPE plates may sometimes be blocked by proteins or gel debris from in-gel protein digestion, resulting in raised back pressure and making manual operation more difficult or even- tually dis-operational. In contrast to off-probe SPE sample purification methods, the direct enrichment and detection of protein/peptides on a matrix-assisted laser desorption/ ionization (MALDI) target is known to improve MALDItime of flight (TOF) sensitivity due to minimal sample loss from fewer sample-handling steps and is usually free of clotting problems. Boronic acid-functionalized gold-coated silica wafer, ${ }^{6}$ ) gold nanoparticles (AuNPs)-sintered on the MALDI plate, ${ }^{7)}$ and boronic acid-modified gold microspot ${ }^{8)}$ have been developed for on-probe MALDI-TOF analysis of glycopeptides or glycoproteins. Recently, we have developed a rapid approach for fabricating functionalized plates through bonding silica-based functionalized particles on polydimethylsiloxane (PDMS) membrane. ${ }^{9)}$ Herein, a novel approach for on-plate selective enrichment and purification of glycoproteins/glycopeptides is proposed based on the new boronic-funtionalized MALDI plate. This approach demonstrates the potential applications in the rapid analysis of glycoproteins/glycopeptides in biological samples.

\section{MATERIALS AND METHODS}

PDMS prepolymer was purchased from Dow Corning (Sylgard 184, Midland, MI, USA). Acetonitrile (ACN) and formic acid (FA) were purchased from J.T. Baker (Phillipsburg, NJ, USA). Dithiothreitol (DTT), iodoacetamide (IAA), horseradish peroxidase (HRP), bovine serum albumin (BSA), and trifluoroacetic acid (TFA) were from Sigma-

\footnotetext{
* Correspondence to: Chao-Jung Chen, Graduate Institute of Integrated Medicine, China Medical University, Taichung 40402, Taiwan, e-mail: cjchen@mail.cmu.edu.tw
} 
Aldrich (St. Louis, MO, USA). $\mathrm{NH}_{2}$-spherical porous silica particles $(5 \mu \mathrm{m}, 100 \AA$, Develosil) were purchased from Nomura Chemical Co., Ltd. (Seto, Japan). Trypsin (modified, sequencing grade) was from Promega (Madison, WI, USA). Sinapic acid (SA) and 2,5-dihydroxybenzoic acid (DHB) were purchased from Bruker Daltonics (Germany). Deionized water (Milli-Q, Millipore, USA) was used to prepare the sample and matrix solutions.

\section{Synthesis of boronic acid particles}

The amine-functionalized silicate particles $(10 \mathrm{mg})$ were suspended with $0.5 \mathrm{~mL}$ of ethanol. 4-Formylphenylboronic acid solution $(40 \mathrm{mg}$ ) was added into the particle solution. After vibration for $48 \mathrm{~h}$ at $50^{\circ} \mathrm{C}, \mathrm{NaBH}_{3} \mathrm{CN}(5 \mathrm{mg}$ in $0.4 \mathrm{~mL}$ ethanol) was added in the particle solution. After reaction for $24 \mathrm{~h}$ under stirring, the particles were collected and washed with $50 \mathrm{mM}$ ammonia bicarbonate solution.

\section{Protein digestion}

HRP or BSA was dissolved in $50 \mathrm{mM}$ ammonium bicarbonate buffer (ABC buffer) and heated to $90^{\circ} \mathrm{C}$ for $20 \mathrm{~min}$. The denatured proteins were reduced with $10 \mathrm{mM}$ DTT for $20 \mathrm{~min}$ at $55^{\circ} \mathrm{C}$, followed by alkylation with $55 \mathrm{mM}$ IAA for $30 \mathrm{~min}$ in the dark at $25^{\circ} \mathrm{C}$. Trypsin was used to digest proteins at an enzyme-to-substrate ratio of $1: 40(\mathrm{w} / \mathrm{w})$ for $12 \mathrm{~h}$ at $37^{\circ} \mathrm{C}$. The peptide sample solution was dried in a centrifugal concentrator (miVac Duo Concentrator; Genevac, Stone Ridge, NY, USA).

\section{Fabrication of boronic acid-coated PDMS plate (BP plate)}

The fabrication of PDMS-coated plate has been reported in our previous studies. ${ }^{9-11)}$ Briefly, an aliquot $(3-5 \mu \mathrm{L})$ of the PDMS mixture (prepolymer:curing agent, 10:1 in volume ratio) was smeared on a glass or a stainless steel MALDI plate and was flatten by a roller (part No. 165-1279; Bio-Rad). After the incubation of the PDMS-coated plate in an oven for polymerization at $80^{\circ} \mathrm{C}$ for $1 \mathrm{~h}$, the plate was washed with a $0.1 \%$ FA solution to remove incompletely polymerized monomers. Aliquots $(3 \mu \mathrm{L})$ of a particle solution $(0.1 \mathrm{mg}$ boronic acid particles in $800 \mu \mathrm{L}, 50 \% \mathrm{MeOH})$ were deposited on a PDMS-coated plate to make the spot arrays. The plate was then incubated in oven at $80^{\circ} \mathrm{C}$ for $1 \mathrm{~h}$, after which it was flushed with $100 \%$ ethanol to remove the excess particles. The boronic acid-coated PDMS plate (BP plate) was ready for use.

\section{Glycopeptide enrichment by boronic acid-coated PDMS plate (BP plate)}

Boronic acid particle spot arrays on the BP plate were first washed with water to remove contaminants and nonspecifically adsorbed compounds. HRP digested peptides (10 ng) or HRP proteins dissolved in $20 \mathrm{mM}$ tris- $\mathrm{HCl}$ ( $\mathrm{pH} 9.5$ ) were loaded onto the boronic acid spots. After incubation, the remaining solution was removed, and the spots were covered with $1.5 \mu \mathrm{L}$ of a DHB matrix solution $(5 \mathrm{mg} / \mathrm{mL}$ in $30 \%$ ACN/0.1\% TFA), followed by MALDI-TOF analysis.

\section{MALDI-TOF MS analysis}

Glycoproteins and glycopeptides were analyzed by MALDI-TOF/TOF-MS (Ultraflex III TOF/TOF; Bruker Daltonics) equipped with a Smartbeam laser. Peptide mass calibration for MALDI-TOF was performed with a peptide calibration standard kit (Bruker Daltonics) to calibrate a mass range of $800-4000 \mathrm{Da}$. A protein calibration standard 1 (Bruker Daltonics) containing insulin, ubiquitin I, cytochrome $c$, myoglobin was used to calibrate a mass range of 4000-30000 Da. For peptide/glycopeptide analysis, spectra were acquired in reflector mode. For protein analysis, spectra were acquired in linear mode with 100 ns pulsed ion extraction time. Mass spectra were processed by flexAnalysis software (Bruker Daltonics).

\section{RESULTS AND DISCUSSION}

Syntheses and characterization of boronic acidfunctionalized particles

Boronic acid modification of silica particles was performed using 4-formylphenylboronic acid; the main steps were shown in Scheme 1. Figure 1 shows the FT-IR spectra of aminophenylboronic acid-functionalized particles. The peaks at $1340 \mathrm{~cm}^{-1}$ and $1376 \mathrm{~cm}^{-1}$ are associated with

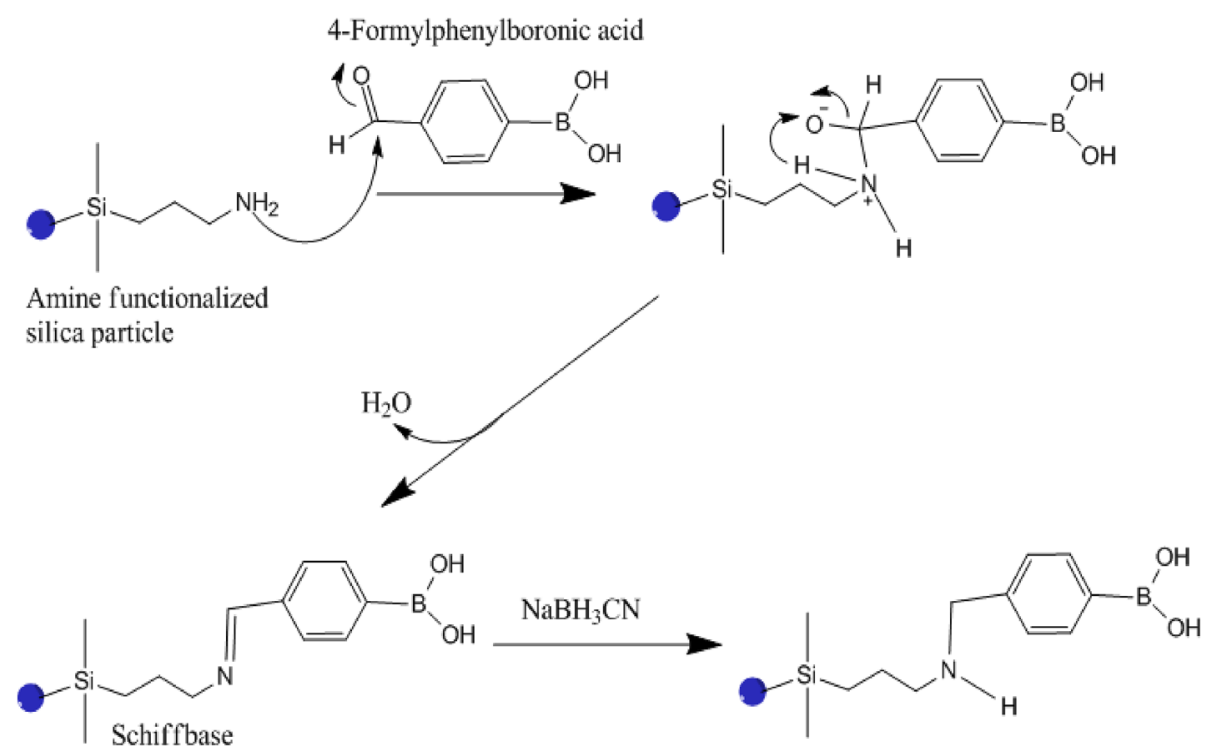

Scheme 1. The synthesis of boronic acid-functionalized silica particle with 4-formylphenylboronic acid. 


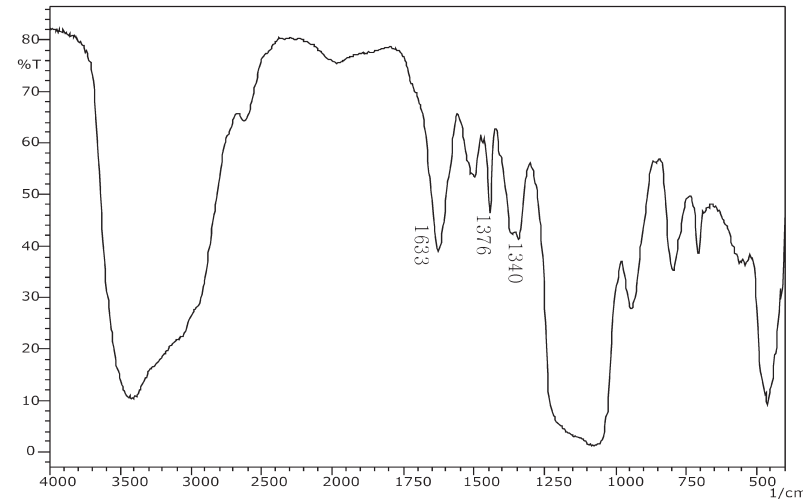

Fig. 1. IR absorption spectra of 4 -formylphenylboronic acid-functionalized particles.

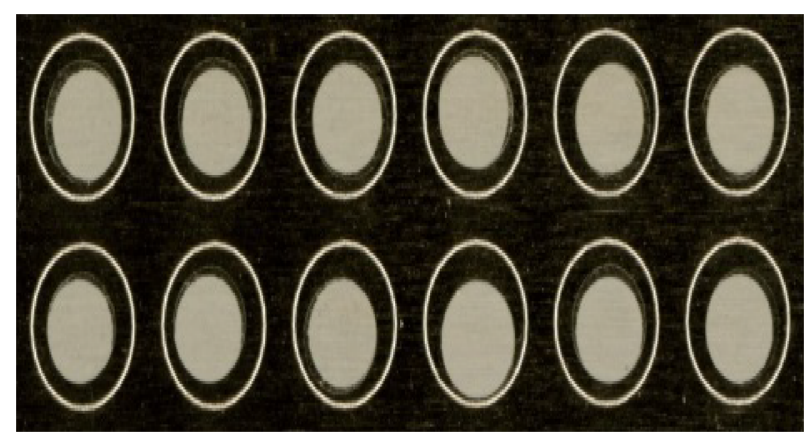

Fig. 2. Spot arrays $(\sim 2.8 \mathrm{~mm}$ i.d.) of boronic acid-functionalized particles on a MALDI-TOF target. The image was obtained using an optical scanner. the $\mathrm{B}-\mathrm{OH}$ and $\mathrm{C}-\mathrm{B}$ vibrations, respectively. The peak at $1633 \mathrm{~cm}^{-1}$ shows the vibrations of phenyl ${ }^{12)}$ and indicates that the silicate particles were successfully functionalized with 4-formylphenylboronic acid groups.

\section{Boronic acid-PDMS plates (BP plate)}

In our previous study, ${ }^{9)}$ we reported that by simply adding silicate particle suspension solution on PDMS, a monolayer of the particles can be immobilized on PDMS film without peeling off after water/organic solvent (ACN or IPA) flushing or sonication due to covalent bonding happen on the contact interface of PDMS and silica particles. In this study, we synthesized boronic acid particles and coated these particles on the PDMS plate. Figure 2 shows that the boronic acid particles can be successfully immobilized on the PDMScoated plate, which can act as the off-line enrichment devices or on-probe detection MALDI plates for glycoprotein/ glycopeptide analysis. The purification flow chart was shown in Fig. 3.

\section{On-probe detection of glycoprotein}

In order to evaluate the sample purification ability on the BP plate, a mixture of BSA (100 ng) and HRP (100 ng) was deposited on the BP plate, followed by wash and elution steps. As shown in Fig. 4a (upper panel), the BSA protein peaks of $66 \mathrm{kDa}$ and $33 \mathrm{kDa}(+2$ charge state) and HRP protein are the major peaks. After BP plate enrichment, only HRP protein peak was observed, and the BSA protein peak was completely removed (Fig. $4 \mathrm{a}$, lower panel). A mixture

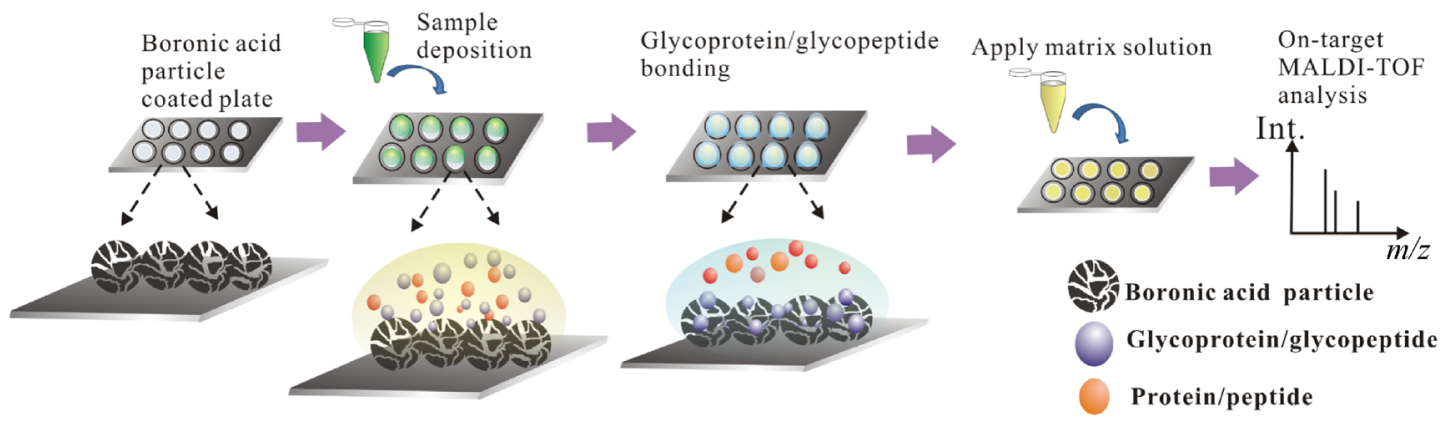

Fig. 3. Schematic representation of the boronic acid-functionalized plate fabrication and analyte enrichment procedures.

(a)

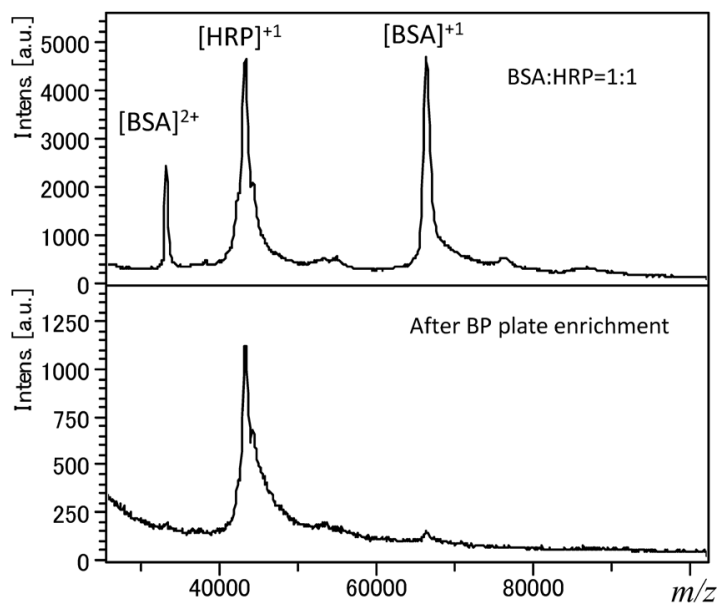

(b)

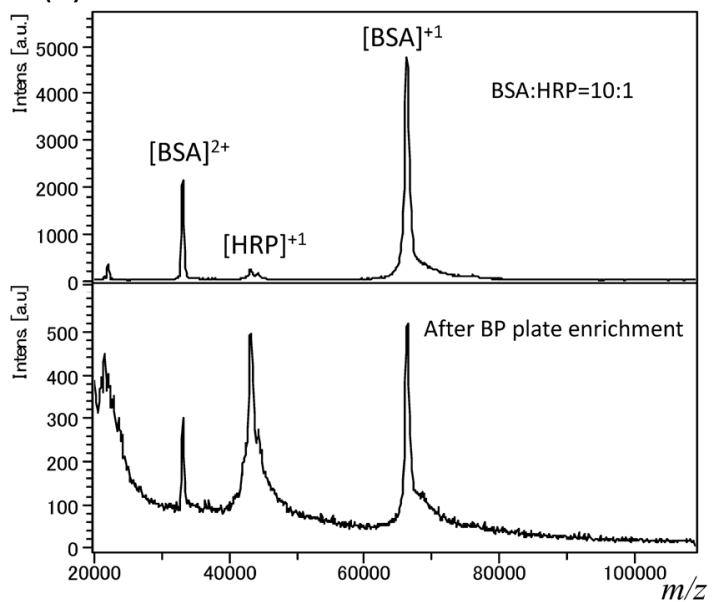

Fig. 4. MALDI-TOF analysis of (a) a mixture of $100 \mathrm{ng}$ BSA / $100 \mathrm{ng}$ HRP and (b) a mixture of $1 \mu \mathrm{g}$ BSA / $100 \mathrm{ng}$ HRP. Upper panel: before BP-plate enrichment. Lower panel: after BP-plate enrichment. 
Table 1. The $N$-glycosylation sites are marked with $\mathrm{N}^{*}$.

\begin{tabular}{cccc}
\hline Peak & $\begin{array}{c}\text { Observed } \\
m / z\end{array}$ & Glycan composition & Amino acid sequence \\
\hline H1 & 2611.2 & Man3GlcNAc2Xyl & MGN $^{*}$ ITPLTGTQGQIR $^{*}$ \\
H2 & 3377.9 & Man2GlcNAc2fuc & GLIQSDQELFSPN ATDTIPLVR $^{*}$ \\
H3 & 3672.1 & Man3GlcNAc2FucXyl & GLIQSDQELFSSPN ATDTIPLVR $^{*}$ \\
H4 & 4222.6 & Man3GlcNAc2FucXyl & QLTPTFYDNSC(AAVESACPR)PN ${ }^{*}$ VSNIVR \\
H5 & 4984.5 & Man3GlcNAc2FucXylMan3GlcNAc2FucXyl & LYN $^{*}$ FSNTGLPDPTLN \\
\hline
\end{tabular}

GIcNAc $=N$-acetylglucosamine, $\mathrm{Fuc}=$ fructose, $\mathrm{Man}=$ mannose, $\mathrm{Xyl}=\mathrm{xylose}$.

(a)

(b)

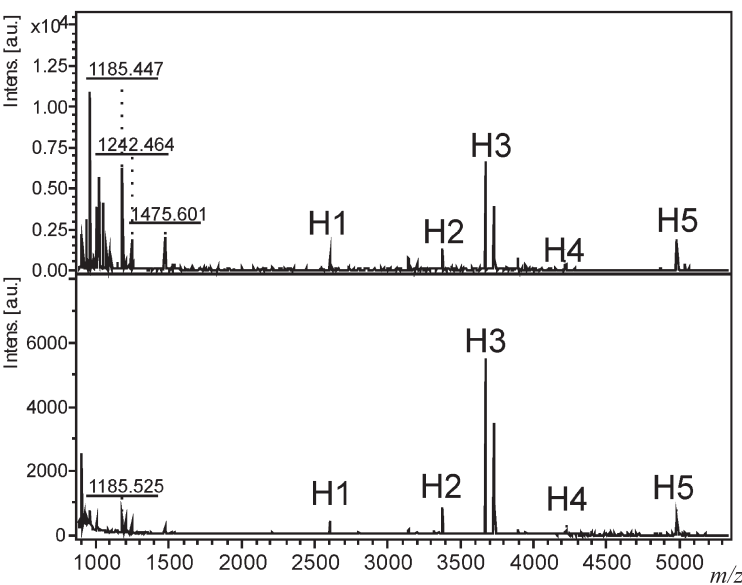

Fig. 5. MALDI mass spectra of a mixture of HRP digested peptides (10 ng). (a) Before BP-plate enrichment; (b) After BP plate enrichment. H1-H5 are glycopeptides of HRP as indicated in Table 1.

of 10 -fold increased protein amount of BSA $(1 \mu \mathrm{g})$ and HRP $(100 \mathrm{ng})$ was also tested. Figure $4 \mathrm{~b}$ (upper panel) shows that HRP is hardly to be detected in the coexistence of 10 fold increased BSA amount. After BP plate enrichment, a significantly improved HRP protein peak signal was observed with significantly reduced BSA protein signals (Fig. $4 \mathrm{~b}$, lower panel). These results indicated the BP plate can effectively purify glycoprotein from high abundant protein mixtures.

\section{On-probe detection of glycopeptides}

In order to evaluate the peptide purification ability on the BP plate, trypsin digested HRP was deposited on the BP plate, after sample incubation for a certain time, the reaming solution was removed, followed by the addition of DHB matrix $(5 \mathrm{mg} / \mathrm{mL}$ in $30 \%$ ACN/0.1\% TFA). Before sample enrichment by BP plate, a mixture of peptide and glycopeptides were detected (Fig. 5a). After applying BP plate, most non-glycopeptides signals in the $\mathrm{m} / \mathrm{z}$ range of $1000-2000$ were significantly reduced leading to a clear spectrum with 5 glycopeptide signals (H1-H5) (Fig. 5b, Table 1). However, when the sample complexity was increased by adding BSA digests in the HRP digests sample, the glycopeptides enrichment performance was not satisfied, even using commercialized boronic acid beads (data not shown). The decreased enrichment efficiency of glycopeptides in more complex peptide digests has also been reported by other groups. ${ }^{1,13}$ This observation arise a concern about the inherent limited ability of boronic acid to enrichment of glycopeptides. Recently, we have made a plate coated with hydrophilic interaction chromatography (HILIC) beads, which successfully enrich 10 HRP glycopeptides in a complex peptides samples. ${ }^{9)}$ However, the HILIC plate has a poor ability in the purification of glycoproteins, which may be attributed to the similar hydrophilicity of glycoprotein and proteins when they are dissolved in water solution.

\section{CONCLUSION}

In this study, we have fabricated a boronic acid-functionalized plate to evaluate its performance on the on-probe detection of HRP protein and HRP glycopeptides. The boronic acid plate can efficiency retain HRP protein in BSA/HRP mixtures and HRP glycopeptides in HRP digests; however, the BP plate did not perform well in the purification of glycopeptides in a BSA/HRP protein digests. Boronic acid may still has limited binding efficiency of glycopeptides but seems to be more suitable for binding glycoproteins with multiple glycan structures.

\section{CONFLICT OF INTEREST}

The authors declare that there are no conflicts of interest.

\section{Acknowledgements}

This work was supported by the China Medical University Hospital (DMR-105-090); the Ministry of Science and Technology (MOST 103-2113-M-039-001-MY2), Biosignature project, Academia Sinica, Taiwan, and Taiwan Ministry of Health and Welfare Clinical Trial and Research Center of Excellence (MOHW105-TDU-B-212-133019).

\section{REFERENCES}

1) Y. Wang, M. Liu, L. Xie, C. Fang, H. Xiong, H. Lu. Highly efficient enrichment method for glycopeptide analyses: Using specific and nonspecific nanoparticles synergistically. Anal. Chem. 86: 2057-2064, 2014.

2) J. Hirabayashi. Lectin-based structural glycomics: Glycoproteomics and glycan profiling. Glycoconj. J. 21: 35-40, 2004.

3) D. F. Zielinska, F. Gnad, J. R. Wisniewski, M. Mann. Precision mapping of an in vivo $N$-glycoproteome reveals rigid topological and sequence constraints. Cell 141: 897-907, 2010.

4) Y. Zhang, M. Kuang, L. Zhang, P. Yang, H. Lu. An accessible protocol for solid-phase extraction of $\mathrm{N}$-linked glycopeptides through reductive amination by amine-functionalized magnetic nanoparticles. Anal. Chem. 85: 5535-5541, 2013.

5) M. H. Selman, M. Hemayatkar, A. M. Deelder, M. Wuhrer. Cotton HILIC SPE microtips for microscale purification and enrichment of glycans and glycopeptides. Anal. Chem. 83: 2492-2499, 2011.

6) Y. Xu, L. Zhang, H. Lu, P. Yang. On-plate enrichment of glycopeptides by using boronic acid functionalized gold-coated $\mathrm{Si}$ wafer. Proteomics 10: 1079-1086, 2010.

7) J. Tang, Y. Liu, D. Qi, G. Yao, C. Deng, X. Zhang. On- 
plate-selective enrichment of glycopeptides using boronic acidmodified gold nanoparticles for direct MALDI-QIT-TOF MS analysis. Proteomics 9: 5046-5055, 2009.

8) J. Tang, Y. C. Liu, D. W. Qi, G. P. Yao, C. H. Deng, X. M. Zhang. On-plate-selective enrichment of glycopeptides using boronic acid-modified gold nanoparticles for direct MALDI-QIT-TOF MS analysis. Proteomics 9: 5046-5055, 2009.

9) H. Y. Liao, F. J. Tsai, C. C. Lai, M. C. Tseng, C. Y. Hsu, C. J. Chen. Rapid fabrication of functionalized plates for peptides, glycopeptides and protein purification and mass spectrometry analysis. Analyst (Lond.) 141: 2183-2190, 2016.

10) C. J. Chen, C. C. Lai, M. C. Tseng, Y. C. Liu, Y. H. Liu, L. W. Chiou, F. J. Tsai. A novel titanium dioxide-polydimethylsiloxane plate for phosphopeptide enrichment and mass spectrometry analysis. Anal. Chim. Acta 812: 105-113, 2014.

11) C. J. Chen, C. C. Lai, M. C. Tseng, Y. C. Liu, S. Y. Lin, F. J. Tsai. Simple fabrication of hydrophobic surface target for increased sensitivity and homogeneity in matrix-assisted laser desorption/ ionization time-of-flight mass spectrometry analysis of peptides, phosphopeptides, carbohydrates and proteins. Anal. Chim. Acta 783: 31-38, 2013.

12) G. Yao, H. Zhang, C. Deng, H. Lu, X. Zhang, P. Yang. Facile synthesis of 4-mercaptophenylboronic acid functionalized gold nanoparticles for selective enrichment of glycopeptides. Rapid Commun. Mass Spectrom. 23: 3493-3500, 2009.

13) C. C. Chen, W. C. Su, B. Y. Huang, Y. J. Chen, H. C. Tai, R. P. Obena. Interaction modes and approaches to glycopeptide and glycoprotein enrichment. Analyst (Lond.) 139: 688-704, 2014. 